\title{
Microcarcinoma papilar de tiroides de bajo riesgo, ¿tratar o vigilar?
}

\section{Low-risk papillary thyroid microcarcinoma, treat or surveil?}

\author{
Pedro Gómez-Romero 1*, Coralys G. Abreu-Rosario², Regina Cano-Saldaña ${ }^{3}$ y Diana Hernández-Juárez ${ }^{4}$ \\ 1Departamento de Endocrinología, Hospital Ángeles Pedregal; ²Departamento de Endocrinología, Centro Médico ABC; \\ ${ }^{3}$ Departamento de Medicina Interna, Hospital Ángeles Pedregal; ${ }^{4}$ Departamento de Endocrinología, Instituto Nacional de Ciencias \\ Médicas y Nutrición "Salvador Zubirán". Ciudad de México, México
}

\section{RESUMEN}

Los cambios epidemiológicos del cáncer de tiroides indican un importante aumento de la incidencia del microcarcinoma papilar. Esta tendencia ha originado el replanteamiento del abordaje de dicha variedad. La vigilancia activa, la cual requiere una evaluación y estratificación adecuada de los pacientes, consiste en realizar un seguimiento estrecho del tumor, aplazando el tratamiento hasta que haya evidencia de progresión de la enfermedad. Esta alternativa se fundamenta en el crecimiento lento que presenta este tipo de tumores, en su baja morbimortalidad y en las posibles complicaciones asociadas a un tratamiento quirúrgico. Aunque la evidencia que apoya a la vigilancia activa cada vez es más robusta, se requiere generar ensayos clínicos y un protocolo específico para cada población. El objetivo de este artículo es exponer el fundamento de la vigilancia activa del microcarcinoma papilar de tiroides, sus indicaciones y la evidencia generada hasta el momento.

Palabras clave: Cáncer de tiroides. Bajo riesgo. Vigilancia activa. Microcarcinoma.

\begin{abstract}
The changing epidemiology of thyroid cancer has shown a significant increase in the incidence of papillary microcarcinoma. This trend has led to the rethinking of the treatment of this type of cancer. Active surveillance, which requires proper evaluation and stratification of patients, consists of closely monitoring the tumor, avoiding immediate treatment. This alternative is based on the slow growth of this type of tumors, its low morbidity and mortality, and the possible complications of surgical treatment. Although the evidence supporting active surveillance is becoming increasingly robust, it is necessary to generate clinical trials and a specific protocol for each population. The objective of this article is to present the basis of active surveillance for papillary thyroid microcarcinoma, its indications and the evidence generated so far.
\end{abstract}

Key words: Thyroid cancer. Low risk. Active surveillance. Microcarcinoma.

\section{Correspondencia:}

*Pedro Gómez-Romero

E-mail: rgp1089@gmail.com
Fecha de recepción: 03-08-2020

Fecha de aceptación: 25-08-2020

DOI: 10.24875/RME.20000079
Disponible en internet: 02-06-2021

Rev Mex Endocrinol Metab Nutr. 2021;8:73-82

2462-4144 / @ 2020 Sociedad Mexicana de Nutricion y Endocrinologia, AC. Publicado por Permanyer. Éste es un artículo open access bajo la licencia CC BY-NC-ND (http://creativecommons.org/licenses/by-nc-nd/4.0/). 


\section{INTRODUCCIÓN}

De forma global se estima que el cáncer de tiroides (CT) representa el $2.1 \%$ de todos los cánceres diagnosticados, por lo que es la neoplasia endocrinológica más frecuente ${ }^{1}$. Aunque la mayoría de los pacientes con $\mathrm{CT}$ tienen un pronóstico favorable, esta enfermedad se presenta con un comportamiento heterogéneo, por lo que existen herramientas para estimar el riesgo de recurrencia y la mortalidad de cada caso ${ }^{2,3}$. Adicionalmente, en las últimas décadas se ha observado un rápido aumento de la incidencia del CT. Algunos autores han propuesto que en ciertas poblaciones este comportamiento epidemiológico se explica por un sobrediagnóstico debido al avance tecnológico de los estudios imagenológicos y como resultado de programas de escrutinio poblacional, como es el caso de Corea del Sur ${ }^{4-6}$. Este sobrediagnóstico se entiende como la detección incidental de tumores subclínicos que nunca hubieran causado morbimortalidad si no hubieran sido diagnosticados. Ante este escenario, se inició en 1993 en el Hospital Kuma, Japón, el primer estudio para evaluar el uso de la vigilancia activa (VA) en pacientes con microcarcinoma papilar (MCP) de tiroides ${ }^{7}$. Este método, que ya se utilizaba en pacientes con cáncer de próstata ${ }^{8}$, consiste en realizar un seguimiento del tumor de por vida en lugar de someter al paciente al tratamiento tradicional con cirugía, yodo radioactivo y tratamiento supresivo con hormonas tiroideas, evitando el sobretratamiento de cánceres que probablemente no representarán un riesgo para el paciente durante toda su vida por su lento crecimiento ${ }^{\text {. }}$ Desde entonces ha aumentado la evidencia respecto al uso de la VA en el CT y ha sido causa de debate.

El objetivo de este trabajo es exponer el fundamento de la VA para el cáncer papilar de tiroides (CPT), sus indicaciones y la evidencia generada hasta el momento.

\section{Cambios epidemiológicos del cáncer de tiroides}

Los nódulos tiroideos son comunes en la población general y su prevalencia depende de diversos factores, incluyendo el método con el que son detectados. Su tasa de detección varía del 4 al 7\% mediante la exploración física, hasta alcanzar del 30 al $67 \%$ por ultrasonido ${ }^{10}$. Estas lesiones son benignas en más del $90 \%$ de los casos y, del porcentaje restante, aproximadamente el $85 \%$ corresponden a $\mathrm{CPT}^{11}$.

Como se mencionó previamente, en 1999 el gobierno de Corea del Sur inició un programa de escrutinio de varios tipos de cánceres y otras enfermedades, en el que se podía incluir la realización de un ultrasonido de tiroides por un bajo costo. La incidencia de esta entidad aumentó 15 veces de 1993 a 2011, a expensas de un incremento del CPT y sin observarse cambios significativos de la mortalidad. Más de 40,000 personas fueron diagnosticadas con CT en 2011,100 veces más personas que aquellas que murieron por dicha neoplasia en la década previa (300 a 400 por año). Este tipo de cáncer se convirtió en el más frecuente en ese país, con un incremento del $25 \%$ en la incidencia anual durante la última década ${ }^{4}$.

En EE.UU. también se reportó esta tendencia: de 1975 a 2009 la incidencia aumentó de 4.9 a 14.3 casos por 100,000 personas, con un incremento más marcado en las mujeres. La mitad de los casos se documentaron en los últimos siete años y la mortalidad se reportó estable, con 0.4-0.6 muertes por 100,000 personas $^{5,6}$. De acuerdo con los autores de estas publicaciones esto se debe en gran medida al uso de estudios de imagen que permiten la detección de tumores con diámetro menor a $10 \mathrm{~mm}$. Estos tumores reciben la denominación de MCP por su tamaño, independientemente de la presencia de factores de alto riesgo de mortalidad como parálisis de cuerdas vocales, metástasis ganglionares o a distancia ${ }^{12}$.

Específicamente la incidencia del MCP también ha aumentado. En 1988 representaba el 25\% de todos los CPT, hasta alcanzar el $39 \%$ en el $2009^{5}$. Adicionalmente, los tumores resecados son cada vez más pequeños. En 1995 el 14\% de estos fueron menores a $10 \mathrm{~mm}$ y 10 años después el $56 \%$ presentaba dicho tamaño ${ }^{4}$. Este comportamiento sugiere que existe un reservorio de la enfermedad, entendido como un porcentaje de la población que padece la entidad, pero que no la manifiesta en ningún momento de su vida. Este hecho puede confirmarse con estudios histopatológicos ${ }^{6}$. Dos series de autopsias de pacientes que 
Tabla 1. Características del cáncer papilar de tiroides de bajo riesgo

\begin{tabular}{|c|c|c|}
\hline Bajo riesgo de recurrencia & Bajo riesgo de mortalidad & Muy bajo riesgo \\
\hline $\begin{array}{l}\text { - Sin evidencia de metástasis locales ni } \\
\text { a distancia } \\
\text { - Resección completa del tumor } \\
\text { - Sin evidencia de invasión locorregional } \\
\text { - Sin evidencia de invasión vascular } \\
\text { - Variedad histológica no agresiva } \\
\text { - Si se administró }{ }^{131} \mid \\
\text { - Sin evidencia de captación fuera del } \\
\quad \text { lecho quirúrgico en el primer rastreo } \\
\text { corporal } \\
\text { - CPT con N0 o < } 5 \text { N1 micrometástasis } \\
\text { (<0.2 cm) } \\
\text { - CPT variante folicular encapsulada } \\
\text { intratiroidea } \\
\text { - Cáncer folicular con invasión capsular } \\
\text { sin o con mínima invasión vascular } \\
\text { - MCP intratiroideo unifocal o multifocal, } \\
\text { incluyendo mutación en BRAF V600E }\end{array}$ & $\begin{array}{l}\text { - }<55 \text { años a diagnóstico } \\
\text { - La ETE menor detectada en el examen } \\
\text { histopatológico no repercute en la } \\
\text { categoría T ni en el estadio clínico } \\
\text { - } \text { T3a > } 4 \text { cm confinado a la glándula } \\
\text { tiroides, cualquier N, MO } \\
\text { - T3b de cualquier tamaño con ETE } \\
\text { macroscópica a músculo (ECM, tiroideo } \\
\text { u omohioideo), cualquier N, MO }\end{array}$ & $\begin{array}{l}\text { - MCP sin evidencia de ETE ni metástasis } \\
\text { ganglionares ni a distancia }\end{array}$ \\
\hline
\end{tabular}

CPT: cáncer papilar de tiroides; MCP: microcarcinoma papilar; ETE: extensión extratiroidea; ECM: esternocleidomastoideo.

fallecieron por causas no relacionadas con CT reportaron el hallazgo de CPT en el 22 al 36\% de los especímenes, siendo la mayoría menores a $1 \mathrm{~cm}^{13,14}$.

Estos datos sugieren que el CPT no pone en peligro la vida de la mayoría de los pacientes y motivan a cuestionar la necesidad de someterlos a todos de forma inmediata a tratamiento, teniendo en cuenta los riesgos asociados a este, especialmente los quirúrgicos.

No todos los autores consideran que el sobrediagnóstico es el único componente de estos cambios epidemiológicos. Registros de 1974 a 2013 en EE.UU. documentaron un aumento de la incidencia del CPT de todos los estadios clínicos, incluyendo tumores de más de $4 \mathrm{~cm}$, aunado a un incremento de la mortalidad anual del $1.1 \%{ }^{15}$, por lo que sería recomendable conocer el comportamiento epidemiológico de cada población.

\section{DEFINICIÓN DE MICROCARCINOMA PAPILAR DE BAJO RIESGO}

Para realizar una adecuada estratificación del riesgo de un tumor es necesario recoger información para establecer un pronóstico y así poder elegir la estrategia de tratamiento y seguimiento más apropiada para cada paciente.

La Asociación Americana de Tiroides (ATA) recomienda el sistema de estratificación del American Joint Committee on Cancer/Union for International Cancer Control para establecer el riesgo de mortalidad. Asimismo, la propia organización desarrolló una herramienta para pronosticar el riesgo de recurrencia. Ambas clasificaciones toman en cuenta una serie de características clínicas e histopatológicas para estratificar al paciente ${ }^{2,3}$. EI CT de bajo riesgo se define como aquel tumor con poca probabilidad de recurrir y de causar morbimortalidad. Las características de bajo riesgo se mencionan en la tabla $1^{2,16}$.

Algunos autores han propuesto clasificaciones del MCP con base en la presentación del tumor, ya sea con o sin síntomas. Sugitani planteó tres grupos de pronóstico: tipo I, aquellos diagnosticados de forma incidental en pacientes asintomáticos con riesgo de recurrencia del $3 \%$ y supervivencia específica del $100 \%$ a 10 años; el tipo II representa un MCP con metástasis ganglionares pequeñas y/o o invasión mínima, lo que correspondería a un CPT de bajo riesgo en etapas iniciales, y el tipo III es un MCP con 
Tabla 2. Clasificación de los pacientes con cáncer de tiroides candidatos para vigilancia activa

\begin{tabular}{|c|c|c|c|}
\hline & $\begin{array}{l}\text { Características del paciente } \\
\text { ideal }\end{array}$ & $\begin{array}{l}\text { Características del paciente } \\
\text { apropiado }\end{array}$ & $\begin{array}{l}\text { Características del paciente } \\
\text { inapropiado }\end{array}$ \\
\hline $\begin{array}{l}\text { Características } \\
\text { del paciente }\end{array}$ & $\begin{array}{l}\text { - Edad > } 60 \text { años } \\
\text { - Comorbilidades severas } \\
\text { - Aceptación por parte del paciente } \\
\text { y expectativa de que se apegue a } \\
\text { la vigilancia } \\
\text { - Conocimiento de la probabilidad } \\
\text { de una probable intervención } \\
\text { quirúrgica en el futuro } \\
\text { - Adecuada red de apoyo }\end{array}$ & $\begin{array}{l}\text { - Edad de } 18 \text { a } 59 \text { años } \\
\text { - Deseos de embarazo } \\
\text { - Historia familiar de cáncer tiroideo }\end{array}$ & $\begin{array}{l}\text { - Edad menor de } 18 \text { años } \\
\text { - Poco cooperador } \\
\text { - Preocupación o negación por } \\
\text { parte del paciente ante el } \\
\text { seguimiento }\end{array}$ \\
\hline $\begin{array}{l}\text { Características } \\
\text { del tumor }\end{array}$ & $\begin{array}{l}\text { - Nódulo tiroideo único } \\
\text { - Bordes bien delimitados } \\
\text { - Sin crecimiento del nódulo en } \\
\text { comparación con algún estudio } \\
\text { previo } \\
\text { - Estadio clínico NO y Mo } \\
\text { - Parénquima sano de al menos } 2 \\
\text { cm alrededor del nódulo } \\
\text { - Sin extensión extratiroidea }\end{array}$ & $\begin{array}{l}\text { - Tumor tiroideo multifocal } \\
\text { - Tumor tiroideo no circunscrito } \\
\text { - Localización subcapsular no } \\
\text { adyacente a estructuras críticas } \\
\text { (tráquea o nervio laríngeo } \\
\text { recurrente) } \\
\text { - Márgenes del tumor no exentos } \\
\text { de enfermedad o extensión } \\
\text { multifocal } \\
\text { - Antecedente de hallazgos } \\
\text { ultrasonográficos inespecíficos que } \\
\text { puedan dificultar el seguimiento } \\
\text { - Microcarcinoma papilar con avidez } \\
\text { por fluorodesoxiglucosa }\end{array}$ & $\begin{array}{l}\text { - Evidencia de histología agresiva en } \\
\text { biopsia por aspiración con aguja } \\
\text { fina } \\
\text { - Localización subcapsular } \\
\text { adyacente a estructuras críticas } \\
\text { - Evidencia clínica de invasión a } \\
\text { estructuras críticas } \\
\text { - Crecimiento } \geq 3 \mathrm{~mm} \\
\text { - Presencia de extensión } \\
\text { extratiroidea } \\
\text { - Estadio clínico N1 o M1 }\end{array}$ \\
\hline $\begin{array}{l}\text { Características } \\
\text { del equipo de } \\
\text { salud }\end{array}$ & $\begin{array}{l}\text { - Disponibilidad de un ultrasonido } \\
\text { de alta resolución } \\
\text { - Equipo multidisciplinario } \\
\text { experimentado en la vigilancia } \\
\text { activa } \\
\text { - Contexto de un ensayo clínico } \\
\text { prospectivo } \\
\text { - Sistema para localizar a los } \\
\text { pacientes y garantizar la vigilancia }\end{array}$ & $\begin{array}{l}\text { - Disponibilidad de un ultrasonido } \\
\text { de forma habitual } \\
\text { - Cirujano endocrinólogo o de cuello } \\
\text { experimentado }\end{array}$ & $\begin{array}{l}\text { - Equipo médico sin experiencia } \\
\text { - Poca disponibilidad de un } \\
\text { oltrasonido de forma habitual }\end{array}$ \\
\hline
\end{tabular}

metástasis ganglionares o parálisis de cuerdas vocales con riesgo de recurrencia del $30 \%$ y supervivencia específica del $74.1 \%{ }^{17}$. Otra propuesta sugiere la división de los MCP según su forma de diagnóstico, ya sea incidental o no ${ }^{18}$.

\section{¿QUIÉN ES CANDIDATO PARA UNA VIGILANCIA ACTIVA Y CÓMO SE LLEVA A CABO?}

La decisión para llevar a cabo esta modalidad gira en torno a ciertas características que debe cumplir un paciente con CPT. Las guías de CT de la ATA del 2015 consideran a la VA como una alternativa a la cirugía inmediata en aquellos pacientes con tumores de muy bajo riesgo, aunque no brindaban recomendaciones muy específicas. En estas guías se consideran candidatos a aquellos que cuentan con una esperanza de vida reducida, cuyas comorbilidades conlleven un elevado riesgo quirúrgico o que dichas comorbilidades deban resolverse antes de que se lleve a cabo el procedimiento quirúrgico ${ }^{2}$. Poco después se publicó un documento por Brito, et al. en el cual se propone agrupar a los posibles candidatos a VA en tres categorías: ideal, apropiado e inapropiado ${ }^{19}$. Para clasificar a un paciente en 
alguno de estos grupos se deben evaluar las características del tumor, las particularidades propias del paciente y la experiencia y recursos del equipo de salud que lo atenderá. Estas categorías se resumen en la tabla $2^{19}$.

De los pacientes considerados como candidatos ideales, únicamente del 1 al $2 \%$ presentará progresión del tumor. Aproximadamente el $10 \%$ de los candidatos apropiados progresará. La VA está contraindicada en el grupo considerado como inapropiado, ya que se ha demostrado que la progresión de la enfermedad en estos pacientes podría causar mayor morbilidad, como en el caso de presencia de extensión extratiroidea o localización subcapsular que conlleve la invasión del nervio laríngeo recurrente o la tráquea ${ }^{16,19}$.

Una vez que se toma la decisión de llevar a cabo una VA, se debe documentar un consentimiento informado en donde se especifiquen de manera clara los beneficios y riesgos de esta estrategia. El seguimiento se llevará a cabo mediante la medición del tumor por ultrasonido. Existen dos protocolos propuestos: el Memorial Sloan-Kettering Cancer Center recomienda una valoración por ultrasonido cada seis meses durante dos años y después de manera anual o bianual ${ }^{19}$; la otra modalidad, propuesta por el Hospital Kuma, consiste en realizar un seguimiento por ultrasonido seis meses después del diagnóstico $y$, si no hay cambios en el tamaño del tumor, anual o bianualmente ${ }^{20}$.

Estos pacientes no serán sometidos a cirugía hasta que se evidencie progresión de la enfermedad. Los criterios de progresión actualmente no son uniformes, sin embargo los usados en el Hospital Kuma son: incremento del tamaño del tumor $>3 \mathrm{~mm}$, evidencia de nuevas metástasis a ganglios cervicales y/o que el tumor alcance un tamaño de $12 \mathrm{~mm}^{20}$. En otros estudios, en los que se han incluido a pacientes con tumores $<15 \mathrm{~mm}$, este último criterio se ha modificado, considerando progresión cuando el tumor alcanza un tamaño $>15 \mathrm{~mm}^{21,22}$. Aunque previamente no se recomendaba la medición de tiroglobulina ( $\mathrm{Tg}$ ) para el seguimiento de estos pacientes ${ }^{19}$, algunos autores también han considerado como criterio de progresión un tiempo de duplicación de Tg menor a un año 21,22 .
PROGRESIÓN DEL CÁNCER DE TIROIDES DURANTE LA VIGILANCIA ACTIVA

La VA se fundamenta en estudios con resultados prometedores. El primero en el que se evaluó este método en pacientes con MCP, mencionado previamente, incluyó a 162 pacientes. Durante un seguimiento de 18 a 113 meses, más del $70 \%$ de los tumores mantuvo un tamaño estable o disminuyó de dimensiones, el $10 \%$ aumentó más de $10 \mathrm{~mm}$ de tamaño y solo se documentó la presencia de metástasis en ganglios de compartimentos laterales en el $1.2 \%^{7}$. En el Cancer Institute Hospital en Tokio se realizó un estudio de vigilancia que incluyó a 230 pacientes con 300 lesiones. El 7\% de los tumores incrementó de tamaño, el 3\% disminuyó y el $90 \%$ restante permaneció sin cambios. Tres pacientes (1\%) presentaron metástasis ganglionares y nueve pacientes (4\%) en los que se detectó aumento del tamaño del tumor recibieron tratamiento quirúrgico durante el periodo de seguimiento (1 a 12 años). Ningún paciente presentó metástasis a distancia o invasión extratiroidea. La supervivencia a 10 años de pacientes sintomáticos con CPT fue del $80 \%{ }^{17}$. En un estudio similar se dio seguimiento a 1,235 pacientes asintomáticos con MCP. Los resultados muestran que el $8 \%$ de las lesiones aumentaron de tamaño, el 3.8\% presentaron metástasis ganglionares nuevas y el $6.8 \%$ progresaron a enfermedad manifiesta a 10 años $^{23}$. Otros datos que apoyan que el pronóstico de estos tumores suele ser bueno son las estadísticas del Surveillance, Epidemiology, and End Results Program de EE.UU., el cual reporta una supervivencia específica para la enfermedad del $99.9 \%$ a cinco años cuando el tumor se encuentra confinado a la tiroides y del $98 \%$ cuando existen metástasis ganglionares locales ${ }^{24}$.

Uno de los primeros estudios de VA en CT realizado fuera de Japón fue llevado a cabo por Tuttle, et al. en EE.UU. con 291 pacientes con diagnóstico de CPT de bajo riesgo. Cabe destacar que el criterio de inclusión que utilizó este grupo fue un tamaño tumoral $<15 \mathrm{~mm}$. Se observó un crecimiento de $3 \mathrm{~mm}$ o mayor en el 3.8\%, con una incidencia acumulada en dos años del $2.5 \%$ y en cinco años del $12.1 \%$. No se presentaron metástasis ganglionares ni a distancia. 
También se observó que una menor edad al diagnóstico y la categoría de riesgo inicial del tumor se asociaron de manera independiente a la probabilidad de crecimiento tumoral ${ }^{25}$. Otro estudio demostró que el 7\% (de una muestra de 291) de los pacientes con CPT con estadio clínico T1b presentó un aumento del tamaño por encima de $3 \mathrm{~mm}$ durante siete años de seguimiento ${ }^{26}$.

Entre los factores que predicen la progresión del tumor están: una edad al diagnóstico menor de 40 años y el embarazo durante el seguimiento de la enfermedad. Los marcadores moleculares se han utilizado como predictores, pero hasta el momento no hay un perfil molecular validado para contraindicar la VA en pacientes de muy bajo riesgo ${ }^{19}$.

Miyauchi, et al. evaluaron la tasa de progresión de la enfermedad en pacientes en VA. Se encontró que esta variaba según la edad de diagnóstico, desde el $36.9 \%$ en pacientes de la tercera década de la vida, descendiendo hasta el $6.6 \%$ alrededor de los 60 años $y$ el $3.5 \%$ en pacientes con 70 años $^{27}$. En un estudio de Ito, et al. en pacientes con menos de 40 años se observó durante 10 años de seguimiento un crecimiento tumoral mayor a $3 \mathrm{~mm}$ en un $12.1 \%$, metástasis ganglionares en un $16.1 \%$ y un aumento total del tumor mayor de $12 \mathrm{~mm}$ en un $22.5 \%$, comparado con los pacientes mayores de 60 años, cuya progresión fue del $4,0.5$ y $2.5 \%$ respectivamente ${ }^{23}$. Se ha demostrado que las personas de edad adulta con MCP son los mejores candidatos para la VA, ya que hay una menor probabilidad de progresión. Adicionalmente se ha observado que los pacientes que requieren tratamiento en algún momento presentan una adecuada respuesta, sin diferencias en cuanto a recurrencia ni morbimortalidad al compararlos con pacientes que recibieron cirugía inmediatamente ${ }^{28,29}$.

Se realizó un estudio con nueve pacientes embarazadas con diagnóstico previo de MCP en VA, en el cual se las comparó con mujeres de iguales características, pero que no estaban embarazadas. Se observó que hubo progresión de la enfermedad en el $44.4 \%$ (4 de 9 pacientes); sin embargo, después al parto, recibieron tratamiento quirúrgico, con un pronóstico similar entre ambos grupos ${ }^{30}$.

Otro factor de riesgo relacionado con progresión es la elevación sostenida de los niveles de hormona estimulante de la tiroides (TSH) mayores a $2.5 \mathrm{mUl} / \mathrm{l}$ durante la VA. Es necesario generar mayor evidencia al respecto, pero mantener un rango de TSH normal-bajo con levotiroxina durante la VA podría considerarse útil ${ }^{31}$.

En un metaanálisis realizado por Cho, et al. en el 2019, cuyo objetivo fue evaluar el aumento del tamaño del MCP de $3 \mathrm{~mm}$ o más y la detección de metástasis ganglionares, se encontró que únicamente el 5.3\% presentó crecimiento del tumor y en el $1.6 \%$ se identificaron metástasis ganglionar en cinco años de seguimiento ${ }^{32}$.

En un estudio realizado por Fukuoka, et al. se evaluaron las diferencias ultrasonográficas entre MCP progresivos y no progresivos. Se encontró un patrón con disminución de la vascularidad peritumoral y un aumento de la densidad de calcificaciones intratumorales en los tumores no progresivos. Estas características podrían ser de utilidad para decidir la frecuencia de la vigilancia ${ }^{33}$. También se han evaluado hallazgos histopatológicos relacionados con progresión de la enfermedad. Aunque para encontrar estos hallazgos se requiere que el paciente se someta a cirugía, se encontró que un mayor índice de proliferación $\mathrm{Ki}-67$ se relaciona con mayor crecimiento del tumor que la presencia de cuerpos de psammoma en un parénquima tiroideo normal y de metástasis intratiroideas se asocian con el desarrollo de metástasis ganglionares ${ }^{34}$.

DESENLACES AL IMPLEMENTAR VIGILANCIA ACTIVA EN EL CÁNCER DE TIROIDES Y SU COMPARACIÓN CON UN TRATAMIENTO QUIRÚRGICO INMEDIATO

En el 2016, en un estudio realizado por Oda, et al., se demostró que los desenlaces de la VA y la cirugía inmediata fueron excelentes y similares. No obstante, hubo un mayor número de efectos adversos en los pacientes que se sometieron a cirugía inmediata, como parálisis de cuerdas vocales (4.1 vs. $0.6 \%$ ) e hipoparatiroidismo transitorio y permanente (16.7 vs. $2.8 \%$ ) a pesar de ser operados por cirujanos expertos $^{35}$. 
En diferentes estudios se ha observado la ausencia de mortalidad en casos de MCP cuando son diagnosticados sin metástasis locorregionales o a distancia. Los estudios que promueven la VA como opción de abordaje han demostrado que los MCP tienen una baja tasa de recurrencias, que va del 1 al 5\%. Por otro lado, debemos de tener en cuenta que el riesgo de complicaciones quirúrgicas permanentes es por lo menos del 1 al 3\%, incluso en centros con cirujanos expertos ${ }^{36}$. Adicionalmente, los adultos mayores con MCP de bajo riesgo que se someten a tiroidectomía total pueden presentar complicaciones y reingresos que se pueden evitar, especialmente en mujeres y en raza negra ${ }^{37}$.

Los pacientes que presentan metástasis ganglionares de novo durante la vigilancia son una minoría, siendo este hallazgo más frecuente en jóvenes. No se puede afirmar que un tratamiento quirúrgico inmediato hubiera evitado el desarrollo de las metástasis y, en caso de haber optado por cirugía de forma inicial, existe la probabilidad de que estos pacientes posteriormente requirieran un segundo tiempo quirúrgico para resecar estas metástasis. Algunos autores consideran que una cirugía oportuna durante la VA es mejor que dos cirugías ${ }^{28}$.

En general, se ha observado que la cirugía inmediata no tiene mayor beneficio o menor riesgo de recurrencias con respecto a la VA, por lo que se ha concluido que un tratamiento más conservador puede ser seguro para pacientes bien seleccionados con este tipo de CT. Algunos factores predictores de metástasis son: una edad más joven ( $<50$ años), sexo masculino y localización del tumor en el polo superior ${ }^{38}$.

La razón más común de someter a cirugía a pacientes en VA ha sido la ansiedad por la probabilidad de progresión de la enfermedad ${ }^{23,39}$, por lo que la calidad de vida es un problema importante a evaluar. Se estudió este aspecto comparando a pacientes en VA con aquellos que habían sido sometidos a lobectomía. Se encontró que el miedo a la progresión de la enfermedad no mostró diferencias significativas entre estos; en cambio, los que recibieron cirugía sí presentaron problemas de salud relacionados con esta, como síntomas neuromusculares, de garganta, boca y cicatri$\operatorname{ces}^{40}$. Se distribuyó una encuesta que fue contestada por 243 pacientes con CT en VA con la cual se encontró que el $32 \%$ de los pacientes al principio del seguimiento refirieron que la preocupación por la progresión del cáncer afectó de manera variable su estado de ánimo. El 14\% informó que la preocupación afectaba sus actividades diarias, siendo el principal motivo de preocupación la ubicación del cáncer y llegar a requerir una intervención quirúrgica. Sin embargo, con el tiempo, el $60 \%$ de los pacientes refirieron que dicha preocupación disminuyó en comparación con el momento del diagnóstico y se sentían satisfechos con la decisión de una VA de la enfermedad ${ }^{41}$.

\section{COSTO}

En un estudio realizado por Oda, et al. en Japón se compararon los costos de realizar inmediatamente un tratamiento quirúrgico en pacientes con $\mathrm{CT}$ frente a los costos de realizar VA durante 10 años. El tratamiento quirúrgico inmediato resultó 4.1 veces más costoso que la VA ${ }^{42}$. En un análisis realizado en Hong Kong mediante un modelo de árbol de decisiones con un caso hipotético de una mujer de 40 años, se comparó la costo-efectividad de una cirugía temprana frente a un abordaje no quirúrgico después de 20 años. Se observó que en los primeros 16 años la VA representa un ahorro de costos; de los 17 años de seguimiento en adelante disminuye este ahorro, sin embargo continúa siendo costo-efectiva en lo que respecta a una mejor calidad de vida ${ }^{43}$. En otro estudio realizado en Australia se calculó que un tratamiento quirúrgico inicial puede ser menos costoso que realizar una hipotética VA por más de 16.2 años $^{44}$.

Estos hallazgos se deben tomar con reserva en cada población debido a la diferencia de costos y de los sistemas de salud en cada país; son necesarios estudios específicos para cada región.

\section{OTROS ASPECTOS Y PERSPECTIVA DE FUTURO DE LA VIGILANCIA ACTIVA}

La mayoría de la evidencia generada hasta el momento sobre la VA proviene de ensayos realizados en Japón, aunque ya se han publicado estudios 
realizados en países como EE.UU. ${ }^{25}$, Corea ${ }^{39}$ y Argentina ${ }^{21,22}$ con resultados promisorios. Es necesario continuar el desarrollo de evidencia sobre VA en diferentes poblaciones por la heterogeneidad de pacientes, sistemas de salud, aceptación y experiencia médica con esta estrategia.

Actualmente existen cinco estudios registrados en progreso, encontrándose cuatro de ellos en proceso de reclutamiento, en Corea ${ }^{45,46}$, EE.UU. ${ }^{47}$, Canadá ${ }^{48}$ e Italia ${ }^{49}$. Estos nos brindarán información adicional sobre el uso de VA en poblaciones fuera de Japón.

En América Latina, hasta donde llega el conocimiento de los autores, solo se han publicado dos estudios con población argentina, realizados por Smulever y Pitoia ${ }^{21,22}$. En el primer estudio, realizado en 2019 , solo el $25 \%$ de los pacientes candidatos a VA aceptó esta estrategia y el $10 \%$ la abandonó por ansiedad. En un seguimiento promedio de 4.6 años, el $17 \%$ de los pacientes presentó un crecimiento significativo del tumor, pero ninguno presentó evidencia de metástasis ganglionar $\mathrm{o}$ a distancia ${ }^{21}$. En el segundo estudio, realizado en 2020 , el $24.4 \%$ de los pacientes que eran candidatos para VA pero recibieron un tratamiento quirúrgico inicial presentaron complicaciones asociadas a la cirugía, de los cuales el $9.6 \%$ tuvo eventos adversos permanentes $^{22}$.

La renuencia por parte de médicos y pacientes es otra limitante para la VA. En dos encuestas realizadas en Australia se reportó que ni médicos ni pacientes estaban listos para aceptar un tratamiento no quirúrgico para MCP de bajo riesgo ${ }^{50,51}$. Ito, et al. reportaron en 2018 que, con el paso del tiempo, en Japón esta modalidad ha tenido más resistencia en el entorno de los cirujanos y su aplicación por endocrinólogos ha aumentado. Se observó que la frecuencia de su uso aumentó gradualmente del 30\% entre 1993 y 1997 hasta el $88 \%$ entre 2014 y $2016^{52}$. Se han descrito herramientas, que deben ser validadas en poblaciones específicas, para facilitar la discusión entre el médico y el paciente sobre la posibilidad de realizar VA. Una de ellas es la que desarrollaron Brito y su equipo, la cual pretende exponer la mayor cantidad posible de evidencia con un lenguaje compresible e infografías accesibles para los pacientes ${ }^{53}$. En un estudio realizado por
Nickel, et al. se observó que el utilizar términos como «lesión papilar» o «células anormales» puede disminuir la ansiedad de los pacientes al momento de discutir opciones terapéuticas ${ }^{54}$. Estas estrategias pueden ser de utilidad para aumentar la aceptación de medidas menos invasivas.

\section{CONCLUSIONES}

La mayoría de los pacientes con MCP no muestran un crecimiento tumoral significativo durante largos periodos de observación. Aquellos que presentan progresión de la enfermedad y requieren cirugía durante la VA suelen tener un buen pronóstico. Esta es una alternativa atractiva para el abordaje de pacientes debidamente seleccionados cuyo seguimiento estará a cargo de un equipo experimentado y con recursos suficientes.

En una carta al editor realizada por Haser, et al. se considera que para implementar exitosamente la VA es importante asegurar una continuidad del manejo del paciente, almacenar los datos del ultrasonido de una forma detallada y uniforme, educar a los pacientes y a los médicos sobre los criterios de inclusión y exclusión, realizar un seguimiento de la calidad de vida del paciente durante la VA y realizar estudios sobre los desenlaces de estos pacientes ${ }^{55}$. Recalcamos la importancia de llevar a cabo ensayos clínicos en nuestro medio para valorar tanto la factibilidad de realizar VA en nuestras instituciones, como para analizar el comportamiento clínico de nuestra población al utilizar esta estrategia. En caso de que se considere como una alternativa factible para nuestros centros hospitalarios, también es necesaria la validación y el desarrollo de herramientas que faciliten la comprensión y permitan disminuir la ansiedad de los pacientes al momento de proponer una VA.

También vale la pena mencionar la oportunidad que existe de generar evidencia sobre el uso de otros métodos no invasivos para el tratamiento del MCP de bajo riesgo, como la ablación con láser, radiofrecuencia o con la aplicación de etanol o polidocanol. 
FINANCIAMIENTO

Los autores no recibieron ningún tipo de patrocinio para realizar este artículo.

\section{CONFLICTO DE INTERESES}

Los autores declaran no tener ningún conflicto de intereses.

\section{RESPONSABILIDADES ÉTICAS}

Protección de personas y animales. Los autores declaran que para esta investigación no se ha realizado experimentos en seres humanos ni animales.

Confidencialidad de lo datos. Los autores declaran que en este artículo no aparecen datos de pacientes.

Derecho a la privacidad y consentimiento informado. Los autores declaran que en este artículo no aparecen datos de pacientes.

\section{BIBLIOGRAFÍA}

1. Kitahara CM, Sosa JA. The changing incidence of thyroid cancer. Nat Rev Endocrinol. 2016;12(11):646-53.

2. Haugen BR, Alexander EK, Bible KC, Doherty GM, Mandel SJ, Nikiforov YE, et al. 2015 American Thyroid Association Management Guidelines for Adult Patients with Thyroid Nodules and Differentiated Thyroid Cancer: The American Thyroid Association Guidelines Task Force on Thyroid Nodules and Differentiated Thyroid Cancer. Thyroid. 2016;26(1):1-133.

3. Tuttle RM, Haugen B, Perrier ND. Updated American Joint Committee on Cancer/Tumor-Node-Metastasis Staging System for Differentiated and Anaplastic Thyroid Cancer (Eighth edition): What changed and why? Thyroid. 2017;27(6):751-6.

4. Ahn HS, Kim HJ, Welch HG. Korea's thyroid-cancer "epidemic": screening and overdiagnosis. N Engl J Med. 2014;371:1765-7.

5. Davies L, Welch HG. Current thyroid cancer trends in the United States. JAMA Otolaryngol Head Neck Surg. 2014;140(4):317-22.

6. Brito JP, Davies L. Is there really an increased incidence of thyroid cancer? Curr Opin Endocrinol Diabetes Obes. 2014;21(5):405-8.

7. Ito Y, Uruno T, Nakano K, Takamura Y, Miya A, Kobayashi K, et al. An observation trial without surgical treatment in patients with papillary microcarcinoma of the thyroid. Thyroid. 2003;13(4):381-7.

8. Parker C. Active surveillance: towards a new paradigm in the management of early prostate cancer. Lancet Oncol. 2004;5(2):101-6.

9. Chung KW. Clinical application of active surveillance in papillary thyroid microcarcinoma. Ann Thyroid. 2017; 2(3):7.
10. Singh Ospina N, Iñiguez-Ariza NM, Castro MR. Thyroid nodules: diagnostic evaluation based on thyroid cancer risk assessment. BMJ. 2020;368:16670.

11. Lowenstein LM, Basourakos SP, Williams MD, Troncoso P, Gregg JR Thompson TC, et al. Active surveillance for prostate and thyroid cancers: evolution in clinical paradigms and lessons learned. Nat Rev Clin Oncol. 2019;16(3):168-84.

12. Miyauchi A, Ito $\mathrm{Y}$. Conservative surveillance management of low-risk papillary thyroid microcarcinoma. Endocrinol Metab Clin North Am. 2019;48(1):215-26.

13. Harach HR, Franssila KO, Wasenius VM. Occult papillary carcinoma of the thyroid: a "normal" finding in Finland: a systematic autopsy study. Cancer. 1985;56(3):531-8.

14. Martinez-Tello FJ, Martinez-Cabruja R, Fernandez-Martin J, Lasso-Oria C, Ballestin-Carcavilla C. Occult carcinoma of the thyroid. A systematic autopsy study from Spain of two series performed with two different methods. Cancer. 1993;71(12):4022-9.

15. Lim H, Devesa SS, Sosa JA, Check D, Kitahara CM. Trends in thyroid cancer incidence and mortality in the United States, 1974-2013. JAMA. 2017;317(13):1338-4.

16. Iñiguez-Ariza NM, Brito JP. Management of low-risk papillary thyroid cancer. Endocrinol Metab (Seoul). 2018;33(2):185-94.

17. Sugitani I, Toda K, Yamada K, Yamamoto N, Ikenaga M, Fujimoto Y. Three distinctly different kinds of papillary thyroid microcarcinoma should be recognized: our treatment strategies and outcomes. World J Surg 2010;34(6):1222e1231.

18. Kakudo K, Bychkov A, Bai Y, Li Y, Liu Z, Jung CK. The new 4th edition World Health Organization classification for thyroid tumors, Asian perspectives. Pathol Int. 2018;68(12):641-64.

19. Brito JP, Ito $Y$, Miyauchi A, Tuttle RM. A clinical framework to facilitate risk stratification when considering an active surveillance alternative to immediate biopsy and surgery in papillary microcarcinoma. Thyroid. 2016;26(1):144-9.

20. Ito Y, Miyauchi A, Oda H, Kobayashi K, Kihara M, Miya A. Revisiting lowrisk thyroid papillary microcarcinomas resected without observation: was immediate surgery necessary? World J Surg. 2016;40(3):523-8.

21. Smulever A, Pitoia F. Active surveillance in papillary thyroid carcinoma: not easily accepted but possible in Latin America. Arch. Endocrinol. Metab. 2019;63(5):462-9.

22. Smulever A, Pitoia F. High rate incidence of post-surgical adverse events in patients with low-risk papillary thyroid cancer who did not accept active surveillance. Endocrine. 2020;69(3):587-95.

23. Ito $Y$, Miyauchi A, Kihara M, Higashiyama T, Kobayashi K, Miya A. Patient age is significantly related to the progression of papillary microcarcinoma of the thyroid under observation. Thyroid. 2014;24(1):27-34.

24. Lowenstein LM, Basourakos SP, Williams MD, Troncoso P, Gregg JR, Thompson TC, et al. Active surveillance for prostate and thyroid cancers: evolution in clinical paradigms and lessons learned. Nat Rev Clin Oncol. 2019;16(3):168-84.

25. Tuttle RM, Fagin JA, Minkowitz G, Wong RJ, Roman B, Patel S, et al. Natural history and tumor volume kinetics of papillary thyroid cancers during active surveillance. JAMA Otolaryngol Head Neck Surg. 2017;143(10): 1015-20.

26. Sakai T, Sugitani I, Ebina A, Fukuoka O, Toda $K$, Mitani $H$, et al. Active surveillance for T1bNOMO papillary thyroid carcinoma. Thyroid. 2019;29(1):59-63.

27. Miyauchi $A$, Kudo $T$, Ito $Y$, Oda $H$, Yamamoto $M$, Sasai $H$, et al. Natural history of papillary thyroid microcarcinoma: kinetic analyses on tumor volume during active surveillance and before presentation. Surgery. 2019;165(1):25-30.

28. Ito $Y$, Miyauchi A, Oda H. Low-risk papillary microcarcinoma of the thyroid: A review of active surveillance trials. Eur J Surg Oncol. 2018;44(3):307-15.

29. Koshkina A, Fazelzad R, Sugitani I, Miyauchi A, Thabane L, Goldstein DP, et al. Association of patient age with progression of low-risk papillary thyroid carcinoma under active surveillance: A systematic review and meta-analysis. JAMA Otolaryngol Head Neck Surg. 2020;e200368.

30. Shindo H, Amino N, Ito Y, Kihara M, Kobayashi K, Miya A, et al. Papillary thyroid microcarcinoma might progress during pregnancy. Thyroid. 2014;24(5):840-4.

31. Kim HI, Jang HW, Ahn HS, Ahn S, Park SY, Oh YL, et al. High serum TSH level is associated with progression of papillary thyroid microcarcinoma during active surveillance. J Clin Endocrinol Metab. 2018;103(2):446-51.

32. Cho SJ, Suh CH, Baek JH, Chung SR, Choi YJ, Chung KW, et al. Active surveillance for small papillary thyroid cancer: A systematic review and meta-analysis. Thyroid. 2019;29(10):1399-408.

33. Fukuoka O, Sugitani I, Ebina A, Toda K, Kawabata K, Yamada K. Natural history of asymptomatic papillary thyroid microcarcinoma:Time-dependent changes in calcification and vascularity during active surveillance. World J Surg. 2016;40(3):529-37. 
34. Hirokawa M, Kudo T, Ota H, Suzuki A, Miyauchi A. Pathological characteristics of low-risk papillary thyroid microcarcinoma with progression during active surveillance. Endocr J. 2016;63(9):805-10.

35. Oda H, Miyauchi A, Ito Y, Yoshioka K, Nakayama A, Sasai H, et al. Incidences of unfavorable events in the management of low-risk papillary microcarcinoma of the thyroid by active surveillance versus immediate surgery. 2016;26(1):150-5.

36. Leboulleux S, Tuttle RM, Pacini F, Schlumberger M. Papillary thyroid microcarcinoma: time to shift from surgery to active surveillance? Lancet Diabetes Endocrinol. 2016;4(11):933e942.

37. Zambeli-Ljepović A, Wang F, Dinan MA, Hyslop T, Stang MT, Roman SA et al. Extent of surgery for low-risk thyroid cancer in the elderly: equipoise in survival but not in short-term outcomes. Surgery. 2019;166(5):895-900.

38. Jeon MJ, Kim WG, Chung WK, Baek JH, Kim WB, Shong YK. Active surveillance of papillary thyroid microcarcinoma: Where do we stand? Eur Thyroid J. 2019;8(6):298-306.

39. Kwon H, Oh HS, Kim M, Park S, Jeon MJ, Kim WG, et al. Active surveillance for patients with papillary thyroid microcarcinoma: a single center's experience in Korea. J Clin Endocrinol Metab. 2017;102(6):1917e1925.

40. Jeon MJ, Lee YM, Sung TY, Han M, Shin YW, Kim WG, et al. Quality of life in patients with papillary thyroid microcarcinoma managed by active surveillance or lobectomy: A cross-sectional study. Thyroid. 2019;29(7):956-62.

41. Davies L, Roman BR, Fukushima M, Ito Y, Miyauchi A. Patient experience of thyroid cancer active surveillance in Japan. JAMA Otolaryngol Head Neck Surg. 2019;145(4):363-70.

42. Oda $\mathrm{H}$, Miyauchi $\mathrm{A}$, Ito $\mathrm{Y}$, Sasai $\mathrm{H}$, Masuoka $\mathrm{H}$, Yabuta $\mathrm{T}$, et al. Comparison of the costs of active surveillance and immediate surgery in the management of low-risk papillary microcarcinoma of the thyroid. Endocr J. 2017;64(1):59-64

43. Lang BH, Wong CK. A cost-effectiveness comparison between early surgery and non-surgical approach for incidental papillary thyroid microcarcinoma. Eur J Endocrinol. 2015;173(3):367-75.

44. Lin JF, Jonker PKC, Cunich M, Sidhu SB, Delbridge LW, Glover AR, et al. Surgery alone for papillary thyroid microcarcinoma is less costly and more effective than long term active surveillance. Surgery. 2020;167(1):110-6.

45. Park Y. Active surveillance on papillary thyroid microcarcinoma [Internet]. ClinicalTrials.gov; 19 de octubre de 2016 [fecha de consulta: 9 de julio de 2020]. Disponible en: https://clinicaltrials.gov/ct2/show/ NCT02938702

46. Lim DJ. Active surveillance on papillary thyroid microcarcinoma [Internet]. ClinicalTrials.gov; 2 de noviembre de 2016 [fecha de consulta: 9 de julio de 2020]. Disponible en: https://clinicaltrials.gov/ct2/show/ NCT02952612

47. Ho A. Active surveillance of papillary thyroid microcarcinoma [Internet] ClinicalTrials.gov; 2017 [fecha de consulta: 9 de julio de 2020]. Disponible en: https://clinicaltrials.gov/ct2/show/NCT02609685

48. Sawka AM. Deciding on active surveillance or surgery for primary management of low risk papillary thyroid cancer (AS-PTC) [Internet]. ClinicalTrials.gov; 5 de septiembre de 2017 [fecha de consulta: 9 de julio de 2020]. Disponible en: https://clinicaltrials.gov/ct2/show/NCT03271892

49. Pezzullo L. Active Surveillance Versus Surgery in Thyroid Papillary Microcarcinoma (MITICA) [Internet]. ClinicalTrials.gov; 2 de noviembre de 2016 [fecha de consulta: 9 de julio de 2020]. Disponible en: https://clinicaltrials.gov/ct2/show/NCT02952612

50. Nickel B, Brito JP, Barratt A, Jordan S, Moynihan R, McCaffery K. Clinicians' views on management and terminology for papillary thyroid microcarcinoma: A qualitative study. Thyroid. 2017;27(5):661-71.

51. Nickel B, Brito JP, Moynihan R, Barratt A, Jordan S, McCaffery K. Patients' experiences of diagnosis and management of papillary thyroid microcarcinoma: a qualitative study. BMC Cancer. 2018;18(1):242.

52. Ito $Y$, Miyauchi $A$, Kudo $T$, Oda $H$, Yamamoto $M$, Sasai $H$, et al. Trends in the implementation of active surveillance for low-risk papillary thyroid microcarcinomas at Kuma Hospital: gradual increase and heterogeneity in the acceptance of this new management option. Thyroid. 2018;28(4):488e495.

53. Brito JP, Moon JH, Zeuren R, Kong SH, Kim YG, Iñiguez-Ariza NM, et al. Thyroid cancer treatment choice: A pilot study of a tool to facilitate conversations with patients with papillary microcarcinomas considering treatment options. Thyroid. 2018;28(10):1325-31.

54. Nickel B, Barratt A, McGeechan K, Brito JP, Moynihan R, Howard K, et al. Effect of a change in papillary thyroid cancer terminology on anxiety levels and treatment preferences: A randomized crossover trial. JAMA Otolaryngol Head Neck Surg. 2018;144(10):867-74.

55. Haser GC, Tuttle RM, Urken ML. Challenges of active surveillance protocols for low-risk papillary thyroid microcarcinoma in the United States. Thyroid. 2016;26(7):989-90. 